\title{
Two X-ray bright cataclysmic variables with unusual activities: GZ Cnc and NSV 10934
}

\author{
T. Kato ${ }^{1}$, P. A. Dubovsky ${ }^{2}$, R. Stubbings ${ }^{3}$, M. Simonsen ${ }^{4}$, H. Yamaoka ${ }^{5}$, P. Nelson ${ }^{6}$,
} B. Monard ${ }^{7}$, A. Peaece ${ }^{8}$, and G. Garradd ${ }^{9}$

1 Department of Astronomy, Kyoto University, Kyoto 606-8502, Japan

2 MEDUZA group, Vedecko-kulturne centrum na Orave, 02742 Podbiel 194, Slovakia

319 Greenland Drive, Drouin 3818, Victoria, Australia

446394 Roanne Drive Macomb, MI 48044, USA

5 Faculty of Science, Kyushu University, Fukuoka 810-8560, Japan

${ }^{6}$ RMB 2493, Ellinbank 3820, Australia

7 Bronberg Observatory, PO Box 11426, Tiegerpoort 0056, South Africa

832 Monash Ave, Nedlands, WA 6009, Australia

9 PO Box 157, NSW 2340, Australia

Received 29 August 2002 / Accepted 1 October 2002

\begin{abstract}
We report on a discovery of unexpected activities in two X-ray bright dwarf novae. GZ Cnc showed an anomalous clustering of outbursts in 2002, in contrast to a low outburst frequency in the past record. The activity resembles an increased activity seen in some intermediate polars or candidates. We identified NSV 10934, X-ray selected high-amplitude variable star, as a dwarf nova with unusually rapid decline. The outburst characteristics make NSV 10934 a twin of recently discovered intermediate polar (HT Cam) with dwarf nova-type outbursts. We propose that these activities in X-ray strong dwarf novae may be a previously overlooked manifestation of outburst activities in magnetic cataclysmic variables.
\end{abstract}

Key words. accretion, accretion disks - novae, cataclysmic variables - stars: dwarf novae stars: individual: GZ Cnc, NSV 10934

\section{Introduction}

Cataclysmic variables $(\mathrm{CVs})$ are close binary systems consisting of a white dwarf and a red dwarf secondary transferring matter via the Roche-lobe overflow. Some of CVs show dwarf nova (DN) outbursts, which are believed to be a consequence of the instabilities in accretion disks (Osaki 1996). Outbursts of DNe usually occur semi-regularly. The presence of unusual CVs with DN-like outbursts, which significantly deviate from the canonical picture of $\mathrm{DNe}$, has recently been receiving special attention (Ishioka et al. 2002; Szkody et al. 2002). Some of these objects have been proven to intermediate polars (IPs) (for recent reviews of IPs, see Patterson 1994; Hellier 1996), which contain weakly magnetized white dwarfs. Theoretical attempts have been also made to explain these unusual outburst characters in the presence of a global magnetic field (e.g. Angelini \& Verbunt 1989). We hereby report on the discovery of two X-ray bright DNe, which may be further candidates for these IPs with unusual DN-like outbursts.

Send offprint requests to: $\mathrm{T}$. Kato,

e-mail: tkato@kusastro.kyoto-u.ac.jp

\section{GZ Cnc}

GZ Cnc was discovered as a variable star by Takamizawa (Tmz V34). Subsequent observations revealed that this object is a dwarf nova which is identified with a ROSAT source (Kato et al. 2001). The object was independently confirmed to be a cataclysmic variable in the course of optical identifications of ROSAT bright sources (Bade et al. 1998). Jiang et al. (2000) reported an optical spectrum which showed strong Balmer and HeI emission lines. Although Jiang et al. (2000) did not explicitly mentioned, HeII emission lines were detected stronger than in typical dwarf novae (Williams 1983).

Kato et al. (2001) obtained time-resolved CCD photometry of the 2000 February long outburst. The long duration of the outburst and the slow rising rate suggested that GZ Cnc is a good candidate for a long-period dwarf nova. As reported in Kato et al. (2001), the recorded outbursts up to 2001 were relatively rare. Although there were unavoidable seasonal observational gaps, only three outbursts were recorded between 1999 and 2001.

In 2002 March-May, we noticed a dramatic increase of the outburst frequency. Figure 1 shows the light curve of the 2002 


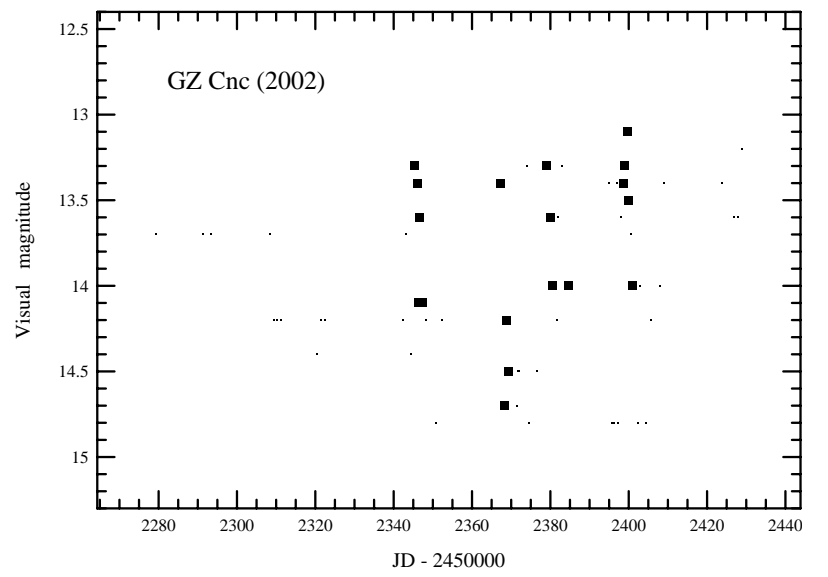

Fig. 1. Light curve of GZ Cnc in the 2002 season. Large and small dots represent positive and negative (upper limit) observations, respectively. Note the high frequency of outbursts (large dots).

season, mainly drawn from visual observations by the authors. Some additional observations (visual and CCD) reported to the VSNET Collaboration ${ }^{1}$ have been incorporated. All observers used $V$-band calibrated comparison stars. The uncertainties of the observations are $0.2-0.3 \mathrm{mag}$, which will not affect the following discussion.

Table 1 lists the known outbursts of GZ Cnc since the discovery by Takamizawa. The shortest interval outbursts in the 2002 unusually active season was only $11 \mathrm{~d}$, and the other two intervals were 21-22 d. As shown in Fig. 1, the durations of the outbursts in 2002 were very short in contrast to the long outburst in 2000 February (Kato et al. 2001). Although such bimodal activity may suggest an outburst activity seen in SU UMa-type dwarf novae (Warner 1985), the apparent lack of superhumps during the long outburst seems to exclude the possibility of an SU UMa-type dwarf nova (Kato et al. 2001).

Alternately, the present behavior in some aspects resembles a "clustering" of outbursts observed in some IPs (EX Hya: Hellier et al. 1989; TV Col: Uemura et al. in preparation), whose interpretation is still in debate (Hellier et al. 2000). Although no clear coherent pulses were detected during the 2000 February outburst (Kato et al. 2001), the presence of HeII emission lines and the relatively strong, hard X-ray spectrum (Bade et al. 1998) makes some resemblance to IPs.

The present activity can also be comparable to V426 Oph, another dwarf nova which is known to show occasionally increased activities (Fig. 2; see also Wenzel \& Splittgerber 1990). V426 Oph has been also suggested to be an IP (Szkody 1986), although this possibility is recently questioned (Hellier et al. 1990). The relatively hard X-ray spectrum of V426 Oph (Verbunt et al. 1997) is also suggestive of an analogy between GZ Cnc and V426 Oph, which may comprise a new class of cataclysmic variables with prominent occasional increases of outburst activities. These activities may be a result of the weak presence magnetic fields, although the evidence of the magnetic nature (at least in V426 Oph) is still tantalizing.

\footnotetext{
${ }^{1}$ http://www. kusastro.kyoto-u.ac.jp/vsnet/
}

Table 1. Outbursts of GZ Cnc.

\begin{tabular}{ccccc}
\hline \hline & Date & & JD-2 400 000 & Max \\
\hline 1994 & November & 30 & 49687 & $13.1^{a}$ \\
2000 & February & 3 & 51578 & $13.7^{b}$ \\
2000 & December & 29 & 51908 & 13.1 \\
2002 & March & 11 & 52345 & 13.3 \\
2002 & April & 2 & 52367 & 13.4 \\
2002 & April & 14 & 52378 & 13.3 \\
2002 & May & 4 & 52399 & 13.1 \\
\hline
\end{tabular}

${ }^{a}$ Discovery observation by Takamizawa.

${ }^{b}$ Long outburst reported in Kato et al. (2001).

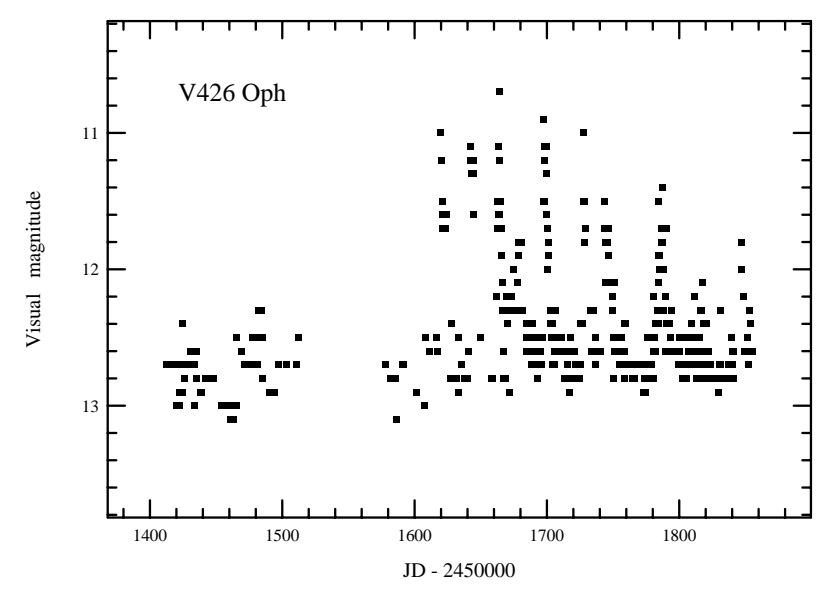

Fig. 2. An state of increased outburst activity in V426 Oph (19992000). The data are from reports to VSNET.

\section{NSV 10934}

NSV 10934 was discovered as a large-amplitude suspected variable star of unknown classification. The cataloged range of variability was 11.2 to $15.0 \mathrm{p}$. We noticed that the object can be identified with a bright ROSAT X-ray source (1RXS J184050.3-834305). Since a combination of a large-amplitude variation and the strong X-ray emission suggests a cataclysmic variable, we started systematic monitoring of this NSV object through the VSNET Collaboration (vsnet-chat 3340$)^{2}$. The first outburst was detected on 2001 March 13 by RS (vsnet-alert 5778$)^{3}$. Three additional outbursts have been recorded since 2002 July. The well-observed most recent two outbursts have been characterized by a sudden rise (more than 1.3 mag within $1 \mathrm{~d}$ ), which established the dwarf nova-type variability. Table 2 lists the observed outbursts. Figure 3 depicts the long-term light curve based on visual observations by the authors (RS, NP, PA) and snapshot CCD observations. The accuracy of the visual observations is $0.2-0.3 \mathrm{mag}$, which will not affect the following discussion. The shortest interval of the observed outbursts is $46 \mathrm{~d}$.

\footnotetext{
${ }^{2}$ http://www.kusastro.kyoto-u.ac.jp/vsnet/Mail/ chat $3000 / \mathrm{msg} 00340 . \mathrm{html}$

${ }^{3}$ http://www.kusastro.kyoto-u.ac.jp/vsnet/Mail/ alert5000/msg00778.html
} 


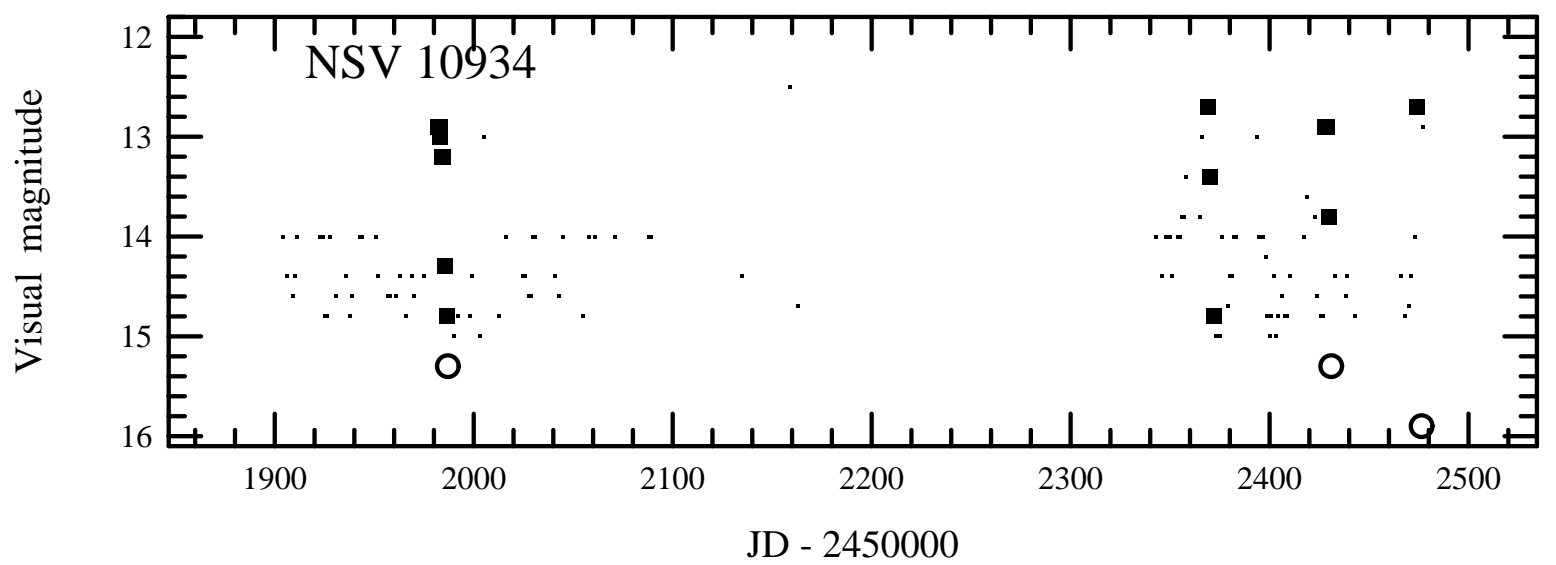

Fig. 3. Long-term light curve of NSV 10934. Large and small dots represent positive and negative (upper limit) observations, respectively. Open circles are unfiltered CCD measurements, which have a sensitivity close to $R_{\mathrm{c}}$. The most recent two CCD observations (NP and NP) have been calibrated by using GSC 9523.1025 (Tycho-2 magnitude: $V=11.73, B-V=+1.07$ ). The overall uncertainty of the CCD photometry is $0.2 \mathrm{mag}$.
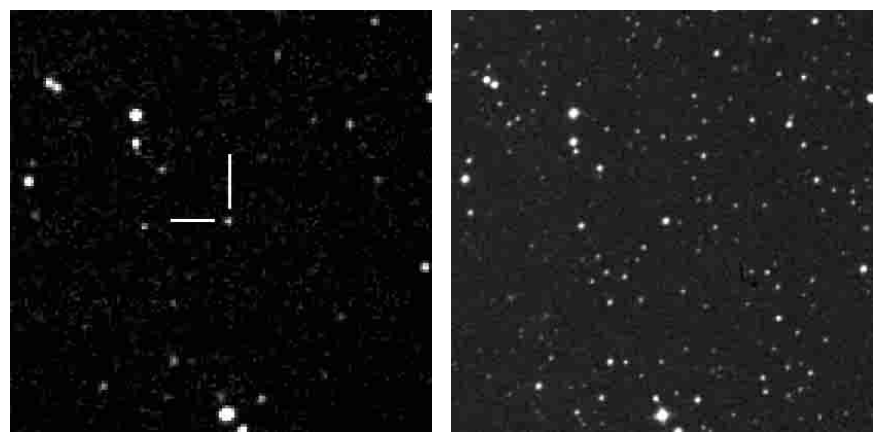

Fig. 4. Identification of NSV 10934. (Left) PN's image on 2002 June 5.423 UT ( 8 arcmin square, north is up, and east is left; magnitude 15.3, slightly above quiescence). (Right) DSS red image showing NSV 10934 in quiescence.

Astrometry of NSV 10934 was performed on CCD images taken by PN (2002 June 5.423 UT) and BM (2002 July $21.024 \mathrm{UT}$ ), both of which were taken during the rapid decline stage from outbursts. The variability of the object has been confirmed by a comparison between the two images. An average of measurements of two images (UCAC1 system, 182 and 71 reference stars respectingly; internal dispersion of the measurements was $\sim 0$ ' $^{\prime} 1$ ) has yielded a position of $18^{\mathrm{h}} 40^{\mathrm{m}} 52^{\mathrm{s}} .52,-83^{\circ} 43^{\prime} 09^{\prime} .84$ (J2000.0). The position agrees with the USNO-A2.0 star at $18^{\mathrm{h}} 40^{\mathrm{m}} 52^{\mathrm{s}} \mathrm{s} 28$, $-83^{\circ} 43^{\prime} 09$ '. 2 (epoch 1983.040 and magnitudes $r=15.6$, $b=16.5$ ), or the GSC-2.2.1 star with position end figures of 52.420 and 09.74 (epoch 1993.767 and magnitudes $r=$ $15.11, b=17.06)$, which is most likely the quiescent counterpart of NSV 10934 (Fig. 4).

Figure 5 shows the enlarged light curve of the best observed outbursts. All the recorded outbursts rather quickly faded. The most recent two outbursts faded more than 1 mag within $3 \mathrm{~d}$ of the outburst maximum. Linear fits to the bestobserved decline stages of the first two outbursts have yielded decline rates of $0.71 \pm 0.06 \mathrm{mag} \mathrm{d}^{-1}$ and $0.71 \pm 0.03 \mathrm{mag} \mathrm{d}^{-1}$, respectively. Rather fragmentary data of the recent two
Table 2. Outbursts of NSV 10934.

\begin{tabular}{lcccc}
\hline \hline & Date & & JD-2 400 000 & Max \\
\hline 2001 & March & 13 & 51982 & 12.9 \\
2002 & April & 4 & 52369 & 12.7 \\
2002 & June & 2 & 52428 & 12.9 \\
2002 & July & 18 & 52474 & 12.7 \\
\hline
\end{tabular}

outbursts further suggest an even higher value close to the termination of the outbursts: the object faded by $1.5 \mathrm{mag}$ in $0.90 \mathrm{~d}$ (JD 2452430.02-.92) and by $3.2 \mathrm{mag}$ in $2.59 \mathrm{~d}$ (JD 2452 473.93-76.52).

The combination of relatively strong and relatively hard (see Table 3) X-ray detection and short optical outbursts either suggests the possibility of an IP with dwarf nova-like outbursts, a non-magnetic HT Cas-like unusual dwarf nova with rather irregularly spaced short outbursts (Kato et al. 2002; Wood et al. 1995), or a system resembling an unusual dwarf nova BZ UMa with short outbursts and quasi-periodic oscillations (Kato 1999; Jurcevic et al. 1994).

The precipitous fading ( $1.5 \mathrm{mag}$ in $0.90 \mathrm{~d})$ recorded during the terminal stage of the outbursts is unlike usual dwarf novae. The sequence of a more slowly fading plateau phase near outburst maxima and a subsequent rapid fading more resembles the behavior of an outburst in the recently discovered IP, HT Cam (Ishioka et al. 2002). The X-ray hardness ratios are also similar (Table 3). HT Cam showed a gradual decline for the first $0.5 \mathrm{~d}$, followed by a dramatic decline by more than $4 \mathrm{mag} \mathrm{d}^{-1}$ (Ishioka et al. 2002). Since the time-evolution of the light curve is slightly slower in NSV 10934, the orbital period of NSV 10934 is expected to be slightly longer than that of HT Cam (86 min), if NSV 10934 indeed turns out to be an HT Cam-like object.

\section{Conclusion}

We report on a discovery of unexpected activities in two X-ray bright dwarf novae. GZ Cnc showed an anomalous clustering 


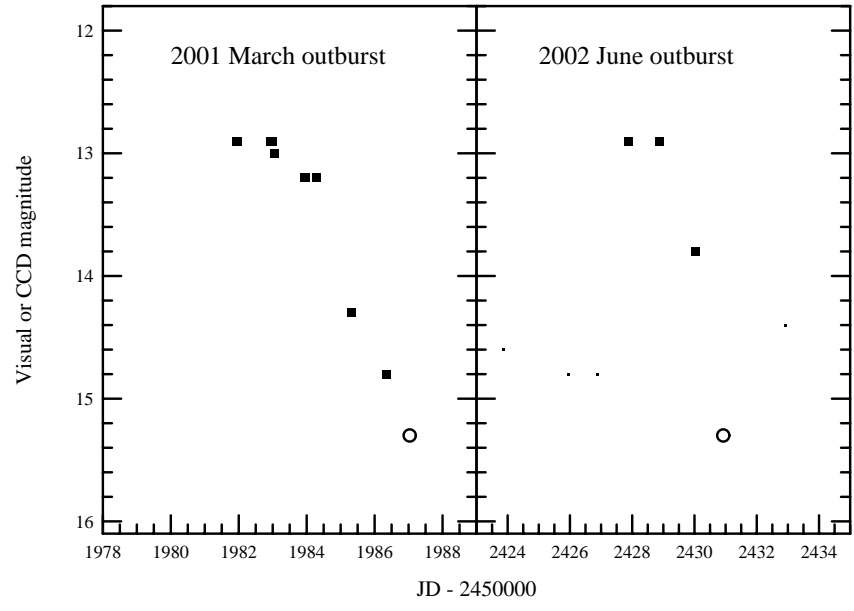

Fig. 5. Enlarged light curve of the best observed outbursts of NSV 10934. The symbols are the same as in Fig. 3.

Table 3. Comparison of X-ray Properties of GZ Cnc, NSV 10934 and $\mathrm{HTCam}{ }^{a}$.

\begin{tabular}{ccccc}
\hline \hline Object & Count rate & HR1 & HR2 & $V$ \\
\hline GZ Cnc & 0.181 & 0.53 & 0.15 & 15.4 \\
NSV 10934 & 0.239 & 1.00 & 0.50 & 15.9 \\
HT Cam & 0.152 & 0.79 & 0.43 & 16.2 \\
\hline
\end{tabular}

${ }^{a}$ The X-ray data are taken from Voges et al. (1999).

of outbursts in 2002, in contrast to a low outburst frequency in the past record. The activity resembles an increased activity seen in some intermediate polars or candidates. We have shown that the outburst characteristics of NSV 10934 closely resembles those of recently discovered intermediate polar (HT Cam) with dwarf nova-type outbursts. The X-ray properties of these objects are summarized in Table 3 . We propose that these activities in X-ray strong dwarf novae may be a previously overlooked manifestation of outburst activities in magnetic cataclysmic variables. Further research to elucidate the relation between these unusual cataclysmic variables and IPs is encouraged. Although direct detection of IP pulses is known to be sometimes difficult or tantalizing (see Rosen et al. (1994) for a recent example) we encourage more extensive search for the IP-type coherent signal in quiescence and outburst (cf. HT Cam: Kemp et al. 2002; Ishioka et al. 2002), and in $\mathrm{X}$-rays.
Acknowledgements. This work is partly supported by a grant-in aid (13640239 (TK), 14740131 (HY)) from the Japanese Ministry of Education, Culture, Sports, Science and Technology. The CCD operation of the Bronberg Observatory is partly sponsored by the Center for Backyard Astrophysics. The CCD operation by PN is on loan from the AAVSO, funded by the Curry Foundation. This research has made use of the Digitized Sky Survey producted by STScI, the ESO Skycat tool, the VizieR catalogue access tool.

\section{References}

Angelini, L., \& Verbunt, F. 1989, MNRAS, 238, 697

Bade, N., Engels, D., Voges, W., et al. 1998, A\&AS, 127, 145

Hellier, C. 1996, in Cataclysmic Variables and Related Objects, ed. A. Evans \& J. H. Wood (Dordrecht: Kluwer Academic Publishers), IAU Colloq., 158, 143

Hellier, C., Kemp, J., Naylor, T., et al. 2000, MNRAS, 313, 703

Hellier, C., Mason, K. O., Smale, A. P., et al. 1989, MNRAS, 238, 1107

Hellier, C., O’Donoghue, D., Buckley, D., \& Norton, A. 1990, MNRAS, 242, 32

Ishioka, R., Kato, T., Uemura, M., et al. 2002, PASJ, 54, 581

Jiang, X. J., Engels, D., Wei, J. Y., Tesch, F., \& Hu, J. Y. 2000, A\&A, 362,263

Jurcevic, J. S., Honeycutt, R. K., Schlegel, E. M., \& Webbink, R. F. 1994, PASP, 106, 481

Kato, T. 1999, Inf. Bull. Variable Stars, 4768

Kato, T., Baba, H., \& Nogami, D. 2002, PASJ, 54, 79

Kato, T., Uemura, M., Buczynski, D., \& Schmeer, P. 2001, Inf. Bull. Variable Stars, 5123

Kemp, J., Patterson, J., Thorstensen, J. R., et al. 2002, PASP, 114, 623

Osaki, Y. 1996, PASP, 108, 39

Patterson, J. 1994, PASP, 106, 209

Rosen, S. R., Clayton, K. L., Osborne, J. P., \& McGale, P. A. 1994 MNRAS, 269, 913

Szkody, P. 1986, ApJ, 301, L29

Szkody, P., Nishikida, K., Erb, D., et al. 2002, AJ, 123, 413

Verbunt, F., Bunk, W. H., Ritter, H., \& Pfeffermann, E. 1997, A\&A, 327,602

Voges, W., Aschenbach, B., Boller, T., et al. 1999, A\&A, 349, 389

Warner, B. 1985, in Interacting Binaries, ed. P. P. Eggelton, \& J. E. Pringle (Dordrecht: D. Reidel Publishing Company), 367

Wenzel, W., \& Splittgerber, E. 1990, Inf. Bull. Variable Stars, 3532

Williams, G. 1983, ApJS, 53, 523

Wood, J. H., Naylor, T., Hassall, B. J. M., \& Ramseyer, T. F. 1995, MNRAS, 273, 772 